\title{
Model and observation of the impact of JTIDS/MIDS on GNSS C/No degradation
}

\author{
Axel Garcia-Pena, Christophe \\ Macabiau \\ École Nationale de l'Aviation Civile \\ Toulouse, France
}

\author{
John Ashley, Dmitri Baraban \\ The MITRE Corporation \\ McLean, Virginia USA
}

\author{
Pierre Durel, Mikael Mabilleau \\ European GNSS Agency \\ Prague, Czech Republic
}

\begin{abstract}
Global Navigation Satellite System (GNSS) L5/E5a interference environment is dominated by DME/TACAN and JTIDS/MIDS pulses causing a degradation of the effective Carrier to Noise $\left(C / N_{0}\right)$ observed by the receiver. This causes delay for the receiver satellite acquisition and impacts the tracking capability. As a mitigation technique, a time-domain blanker is implemented to mitigate their impact. RTCA DO-292 proposes a model to compute the $C / N_{0}$ degradation of the received useful signal by the increase of the noise power spectral density (PSD).
\end{abstract}

This paper developed by ENAC and The MITRE Corporation as part of their cooperative efforts to support RTCA analyses, focus on the impact of Joint Tactical Information Distribution System/Multifunctional Information Distribution System (JTIDS/MIDS) Radio Frequency Interference (RFI) signals. Three relevant interfering scenarios are presented as well as a method to generate time-domain equivalent JTIDS/MIDS signals for interference analysis purposes. Simulated results as well as predicted results are presented for the interfering scenarios. The predicted results are calculated from an updated $C / N_{0}$ degradation formula with respect to RTCA DO-292 proposed formula. The impact of GNSS receiver Radio Frequency Front End (RFFE) filter bandwidth and blanker threshold are evaluated.

Keywords-JTIDS/MIDS, C/N $N_{0}$ degradation, C/N $N_{0}$ effective, $R_{I}$, bdc, Case 8, Case 8A, 50NM APIS, blanker, threshold, correlator

\section{INTRODUCTION}

GNSS received signals processing can be affected by received additive signals such as noise, multipath and interference. RFI sources are of various sorts and their nature and impact depends on the user application. In the context of civil aviation, it is important to identify and characterize the radio frequency interference relevant to the airborne GNSS receivers processing signals in the L1/E1 and L5/E5a bands, to determine the vulnerability of these airborne GNSS receivers equipped with their relevant antenna, to issue minimum requirements on these L1/E1 and L5/E5a antennas, and to set minimum requirements to be imposed on airborne GNSS receivers operating at these bands. Numerous related activities have led to the elaboration of various ICAO, RTCA and EUROCAE standards considering RFI. Currently, [1] reflecting the relevant interference with the L5/E5a frequency band is being updated to incorporate the evolutions of the RFI environment defined by DME/TACAN, JTIDS/MIDS, LDACS, SSR equipment and other GNSS systems operating at these bands, as well as the usage of this L5/E5a band for GALILEO E5a and SBAS L5/E5a datalink airborne signal processing. In addition, the ICAO RFI mask of GNSS L5/E5a receivers is now under definition. These elements will then complement the current draft of EUROCAE and RTCA MOPS for dualfrequency (L1 and L5) and multi-constellation airborne receivers.

The RFI impact on a GNSS receiver in civil aviation is usually modelled as the $C / N_{0}$ degradation observed at the receiver's correlator output, or equivalently, as an increase of the effective $N_{0}$ denoted as $N_{0, \text { eff. }}$ Therefore, a decrease of the minimum available $C / N_{0}$, derived from the link budget and from the $N_{0, \text { eff }}$ calculation, implies a reduction of the $C / N_{0}$ margin between the minimum available $C / N_{0, \text { eff }}$, and the different L5/E5a GNSS and SBAS signal processing, acquisition, tracking, demodulation, $C / N_{0}$ threshold values.

In the course of the elaboration of the update of RTCA DO292 [1], it is proposed to revisit several elements of the worstcase link budget analysis in order to consolidate the overall link budget margin. This was deemed necessary since the link budget margin is expected to be small. Among the axes of revision are:

- the analytical model representing the effect of the Automatic Gain Control/Analog to Digital Converter (AGC/ADC) and temporal blanker.

- the DME/TACAN environment and its impact on proposed performance capabilities for a GNSS L5/E5a receiver. Recent analysis have been conducted in [4].

- the JTIDS/MIDS environment and its impact on proposed performance capabilities for a GNSS L5/E5a receiver.

- the SSR environment and its impact on proposed performance capabilities for a GNSS L5/E5a receiver.

- the newly planned systems operating within the Aeronautical Mobile (en-route) Service [(AM®S] within the $960-1164 \mathrm{MHz}$ band and their impact on proposed performance capabilities for a GNSS L5/E5 receiver. These ${ }^{\circledR}(\mathrm{R}) \mathrm{S}$ systems include the L-band Digital Aeronautical System (LDACS) and Remotely Piloted Aircraft System (RPAS) Command and Control (C2) Data link, and commercial systems authorized in the band in some states such as commercial Programme Making and Special Events (PMSE) equipment.

In order to mitigate the impact of pulsed RFI signals, an airborne GNSS receiver introduces an interference suppression mechanism called a pulse blanker, which has the objective of removing/blanking part of the incoming signal that fulfills a 
certain condition; usually to exceed a set threshold. Various pulsed interference blanking methods have been previously studied ranging from frequency notch filtering, temporal domain blanking and temporal-frequency hybrid filtering [5]-[8]. Traditionally, the countermeasure adopted against pulse interference, which is analyzed in civil aviation is the temporal domain pulse blanking method as described in [1]: to compare the incoming signal envelope power with a threshold and to blank (set to 0 ) the time samples which are above this threshold (issues about its actual implementation are addressed in [2]).

In this paper, the model and observation of the impact of JTIDS/MIDS on GNSS $C / N_{0}$ degradation as a RF pulsed interference is specifically tackled. JTIDS/MIDS stands for Joint Tactical Information Distribution System / Multifunctional Information Distribution System and is a military aeronautical digital tactical communication, navigation and identification system, which is operated on land, sea and airborne platforms in many countries worldwide. More specifically, an update of analysis conducted in [1][9] is done.

The general aim of this paper is first to introduce an equivalent mathematical model for the JTIDS/MIDS signal generation from a $C / N_{0}$ degradation point of view for standardization purposes. More specifically, this paper makes a comparison and clarifies the $R_{I}$ theoretical formulas based on [1] and [3]. Second, this work defines the implementation of an equivalent time domain JTIDS/MIDS signal for interference analysis as well as relevant JTIDS/MIDS interfering scenarios, such as Geographical Area (GA) case 8 [1] and 50NM Any point in Space (APIS). Third, this paper validates the $R_{I}$ and $C / N_{0, \text { eff }}$ theoretical formulas from simplified JTIDS/MIDS simulated signals and scenarios. Finally, prediction and simulation results are given for the most relevant JTIDS/MIDS interfering scenarios.

Note that this article has been jointly authored by ENAC and MITRE. The objective of this joint publication was to demonstrate the benefit of the on-going collaboration between these two institutions in the L5/E5a band interference analysis framework. As a result, this paper contains analysis, results and proposals of both institutions for the timely update of RTCA and EUROCAE minimum GNSS receiver performance requirements.

The paper organization is presented as follows. In Section II, the general $C / N_{0}$ degradation, blanker duty cycle $(b d c)$ and $R_{I}$ analytical expressions are developed after the introduction of a generic airborne civil aviation receiver on the L5/E5a band structure. In this section, a new generic formula for $R_{I}$ developed in [3] is also given. In Section III, the JTIDS/MIDS system and signals are presented as well as the most significant interfering scenarios. In Section IV, the validation of the $R_{I}$ formula proposed in [3] and the understanding of the general $C / N_{O}$ degradation and $b d c$ formulas are made. In Section V, the $C / N_{O}$ degradation results are presented for the most significant interfering scenarios; simulated results, predicted results with customization of $R_{I}$ formula for the JTIDS/MIDS signal are presented. Section VI provides the MITRE Corporation approach and results of the same JTIDS/MIDS modelled interference scenarios. Finally, the analysis is concluded with findings and proposed area for future research.

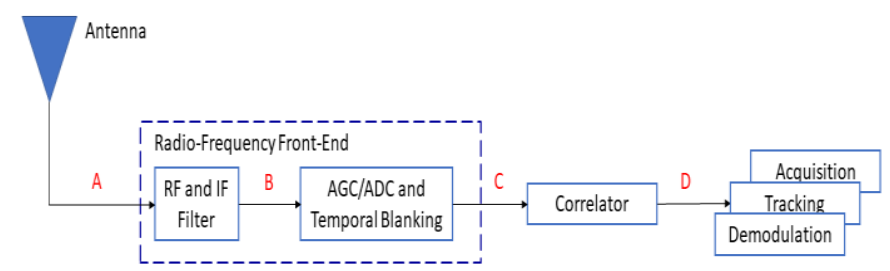

Fig. 1. Generic civil aviation GNSS receiver block scheme

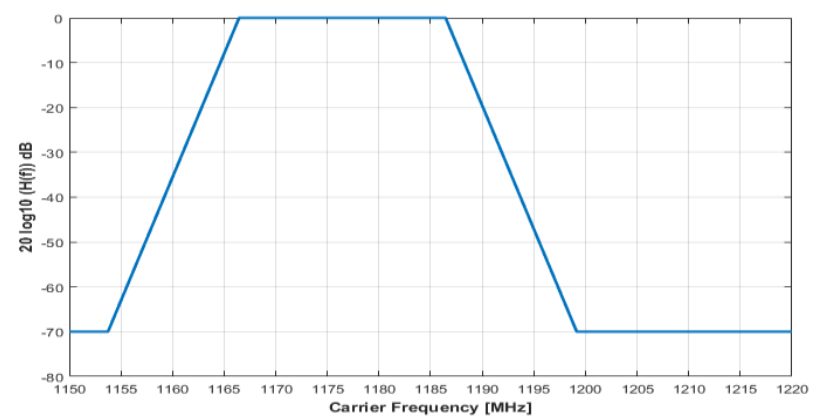

Fig. 2. Radio Frequency Front-End plus antenna equivalent transfer function defined in DO292 customized for a $\mathrm{BW}=20 \mathrm{MHz}$

\section{Understanding of $C / N_{0}$ DEgRADATION ANALYTICAL MODEL}

\section{A. Generic airborne civil aviation GNSS receiver}

In order to understand the $C / N_{0}$ degradation analytical model, a generic airborne civil aviation GNSS receiver structure as well as the behavior and effect of its components on the received signals are described. In Fig. 1, the receiver structure is presented.

First, the antenna is the element responsible of capturing the incoming electro-magnetic waves with modulated signal: at the antenna port (point A), there is a mix of all incoming signals; useful signals, GNSS and SBAS signals, and RFI signals such as DME/TACAN, JTIDS/MIDS, etc. Once the signals have been captured by the antenna, they are passed to the Radio-Frequency Front-End (RFFE) block. This block amplifies the received signals, shifts or down-converts them from their received signal frequency carrier to the intermediate frequency and filters them (removing the image frequency, the spurious frequencies as well as the signal outside the frequency bandwidth of interest). The filtered signals are modelled in Fig. 1 at the RF (RadioFrequency) and IF (Intermediate Frequency) filters output at point B. RTCA DO-292 [1] defines the joint effect of these two filters plus the antenna filtering effect with an equivalent filter transfer function; the equivalent transfer function, $H_{R F}(f)$, for a 20MHz filter bandwidth is provided in Fig. 2.

The RFFE block is also responsible for gain control and digitizing the filtered signals with the application first of the AGC circuit followed by ADC. In the proposed airborne civil aviation L5/E5a GNSS receiver, the digital pulse blanker is introduced after the RFFE block. As explained in the introduction, the blanker is a device, which is going to blank (put to 0 s) the time and/or frequency samples of the incoming signal (mix of signals) that exceed a set threshold; the digitized and post blanker signal is found at point $\mathrm{C}$ of Fig. 1. 


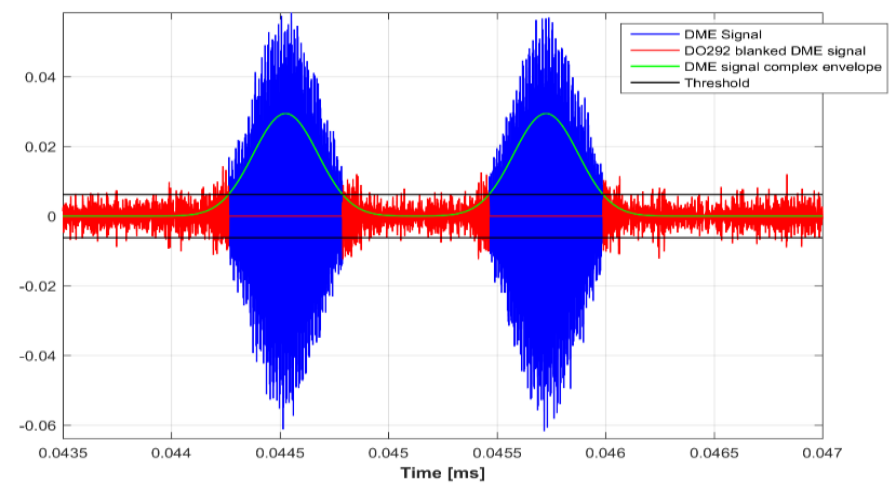

Fig. 3. Example of the behavior of the DO-292 instantaneous blanker over the signal complex envelope

In RTCA DO-292 [1], the defined blanker is a temporal blanker called an "instantaneous blanker". This blanking mechanism removes all the incoming signal time samples, which have a power over a given threshold (issues concerning its actual description and physical implementation are addressed [2]), see Fig. 3. For an optimal functioning, the blanker should also be logically coupled with the AGC/ADC blocks: to ensure that high-power pulses are not saturating the AGC/ADC and that the blanked signal spans the ADC quantization range. While this coupling of the AGC/ADC with the blanker is important, it is out of scope of this paper. The digitized and post blanker signals (point $\mathrm{C}$ of Fig. 1) are fed to the correlator. Finally, the RFI signals are modelled at the correlator output (point D of Fig. 1) where the demodulation, acquisition and tracking capabilities of the receiver can be impacted. It is at this point that these impacts are predicted and simulated within the analysis in this paper.

\section{B. General analytical model}

The key figure of merit to analyze the RFI signals and the blanking method impact is the signal $C / N_{0}$ degradation or more specifically, the difference between the $C / N_{0}$ when only the useful signal is present at the receiver antenna port (no RFI signals) and the $C / N_{O}$ when the useful signal and RFI signals are present at the receiver antenna port (with blanker activation); the latter $C / N_{O}$ is also called effective $C / N_{O}$ or $C / N_{0, e f f}$.

Although the blanking method is going to reduce the average power of the useful signal (part of the information signal is removed as well as the noise power), RTCA DO-292 [1] recommended to model the $C / N_{0, \text { eff }}$ by defining an equivalent $N_{0, \text { eff }}$ while keeping the original useful signal power, $C$. Note that $N_{0, \text { eff }}$ represents the effective noise power spectrum density that a receiver will observe at the correlator output if the receiver captures a useful signal with power $C$ at the correlator output. This assumes that subsequent RFFE elements are considered as ideal (RF filter, IF filter, AGC/ADC), the correlator is also considered ideal, there are no RFI signals present and the blanker is not activated. In other words, in section 2.6.2.3, RTCA DO292 [1] recommended a generic formula to compute the degradation of the $\mathrm{C} / \mathrm{N} 0$ through the increase of the background noise, which is caused by pulsed and continuous RFI. Such computations were based on rigorous evaluation within the RTCA Special Committee 159.

In order to mathematically model $N_{0, e f f}$, the following concepts about the blanking and the incoming signals must be considered. Firstly, although all received L5/E5a GNSS and SBAS signals are by definition useful signals, the receiver has to isolate the signals one-by-one to exploit them. The GNSS and SBAS signals which are not tackled by a specific channel (correlator) are also considered RFI signals. In other words, if the receiver is trying to isolate the GNSS (or SBAS) signal $i$ in one correlator block, all the other GNSS (or SBAS) signals $j, j \neq i$, falling in the L5/E5a band are considered RFI signals. These signals are continuous (non-pulsed) signals and its contribution is modelled with the term $I_{0, W B}$. Note that the blanking method will not target these signals since they are continuous and thus, the blanking method settings will be determined by the pulsed RFI signals, such as DME/TACAN and JTIDS/MIDS. It is important to realize that a blanker threshold must be chosen high enough above the thermal noise and the continuous signal $I_{0, W B}$ to avoid receiver excessive blanking of the useful signal effectively saturating the receiver. Secondly, pulsed RFI signals impacts $C / N_{0, e f f}$ in two different ways:

1) Part of the signal is removed due to the blanking and since the impact on the removed useful signal power, $(1-b d c)^{2}$, is higher than the impact on the power of the noise, $(1-b d c)$, the equivalent $N_{0, \text { eff }}$ can be seen to be increased by a factor of $1 /(1$ $b d c$ ). The acronym $b d c$ represents the blanker duty cycle, or in other words, the percentage of time the incoming useful signal is blanked (bdc $\in[0,1]$ ).

2) Not all the RFI signal samples have a power above the threshold; thus, a part of the RFI signal is not removed and its influence must be added to the thermal noise; $R_{I}$ is the belowthreshold interfering-signal-to-thermal-noise ratio.

From these considerations, $N_{0_{e f f}}$ can be modelled as [1][3]:

$$
\begin{gathered}
N_{0, e f f}=\frac{N_{0}}{1-b d c} \cdot\left(1+\frac{I_{0, W B}}{N_{0}}+R_{I}\right) \\
R_{I}=\sum_{i=1}^{I} R_{I, i}
\end{gathered}
$$

Where $I$ is the total number of pulsed RFI signals,

$R_{I, i}$ is the pulsed source $i$ below-blanker interfering-signalto-thermal-noise ratio, $I_{0, W B}$, also called the $I_{G N S S}$, is the equivalent white noise power spectrum density generated by the continuous interfering signals (in that case only GNSS/SBAS signals are included) at the correlator output.

Finally, $C / N_{O}$ degradation can be calculated by comparing $N_{0, \text { eff }}$ to $N_{0}$ :

$$
D e g=10 \log _{10}\left(1+\frac{I_{0, W B}}{N_{0}}+R_{I}\right)-10 \log _{10}(1-b d c)
$$

\section{DO292 bdc and $R_{I, i}$ Computation}

In RTCA DO-292 [1], $R_{I, i}$ and $b d c$ expressions are found by taking the following assumptions [3]:

1) The blanking mechanism has an effect of uniformly spreading the interfering signal PSD along the RFFE plus antenna equivalent filter bandwidth, $B W$.

2) The useful signal as well as all the interfering signal sources are affected by the same applied blanking duty cycle: 
the same $b d c$ value is applied to the useful signal and to any interfering signals.

3) $b d c$ is computed by assuming no collisions between below-threshold pulses (collisions among below-threshold pulses do not activate the blanker); and by assuming that the blanker activation caused by the different interfering signals follow a time uniform distribution when calculating abovethreshold pulse collisions, in addition to not consider the pulses duration (or blanker activation duration).

RTCA DO-292 [1] customizes the pulsed interfering signals contribution to the $C / N_{0}$ degradation, $R_{I, I}$, as:

$$
\begin{gathered}
R_{I, i}=\beta_{i}\left(\Delta f_{i}\right) \frac{P_{\text {peak }, i}^{\text {out }} d c_{i}}{N_{0} B W} \\
\beta_{i}\left(\Delta f_{i}\right)=\int_{-\infty}^{+\infty}\left|H_{R F}(f)\right|^{2} \bar{S}_{i, B B}\left(f-\Delta f_{i}\right) d f
\end{gathered}
$$

Where $P_{\text {peak }, i}^{\text {out }}$ is the peak power of the pulsed interfering source $i$ at the antenna output or at RFFE input; $d c_{i}$ is the duty cycle of pulsed interference source $i$ that represents the part of the signal power that goes through the blanker so that $P_{\text {Peak }, i}(1-b d c) d c_{i}$ is the post-blanker interfering source signal power; $B W$ is the bandwidth of the equivalent transfer function of the RFFE block plus antenna; $N_{O}$ is the noise power spectrum density; $H_{R F}(f)$ is the baseband transfer function of the equivalent RFFE plus antenna filter; $\bar{S}_{i, B B}(f)$ is the $\mathrm{i}^{\text {th }}$ baseband before-blanker normalized interfering signal PSD; and $\Delta f_{i}$ is approximated as the frequency difference between the $i^{\text {th }}$ interfering signal central frequency and the receiver central frequency, L5 frequency, $\Delta f_{i}=f_{c_{i}}-f_{L 5}$.

RTCA DO-292 [1] customizes the $b d c$ mathematical model as shown below by not considering below-threshold with belowthreshold pulse collisions between the different interfering signals, and by considering a time uniform distribution for interfering source blanker activation for above-threshold with above-threshold pulse collisions in addition to not consider the blanker activation duration. Nevertheless, note that for DME/TACAN signals, a $b d c$ expression is proposed in [1],[9] that encompasses all DME/TACAN pulse collisions.

$$
b d c=1-\prod_{i=1}^{I}\left(1-b d c_{i}\right)
$$

Where $b d c_{i}$ is the blanking duty cycle generated by interfering signal $i$ (all DME/TACAN emitters are modelled as a unique interfering source)

\section{DO292 analytical model limitations}

The limitation of the 3 previously defined assumptions applied in the RTCA DO-292 [1] are described below [3]:

1) The application of the temporal blanking over a signal has as consequence the spreading of the signal PSD. This is because the abrupt nulling of some signal time-domain samples introduces fast variations of the signal amplitude, and thus, high frequency components appear on the post blanker signal PSD. Nevertheless, the assumption of a uniformly spread PSD of the $i^{\text {th }}$ post blanker interfering signal appears to be too conservative.
In fact, as shown in [3], the spreading of the signal is far from transforming the signal into a uniformly spread PSD signal. A priori, this assumption seems to be a worst-case scenario since all interference signals, irrespective of $\Delta f$, are processed equally except for the $\beta_{i}$ term and since their assumed band is larger than the true one.

2) This assumption is fulfilled for all the continuous interfering signals which do not trigger the blanker (below the threshold). However, this assumption may not be fulfilled for the pulsed interfering signals: it is assumed the blanking activation can happen at any moment in time, uniform distribution, and that the RFI pulses below-threshold can be received at any moment in time, uniform distribution. Therefore, if due to the interfering scenario, either the blanker activation (above-threshold RFI pulses arrivals) or the below-threshold RFI pulses arrivals do not follow a uniform distribution, the assumption will lead to an inaccurate $R_{I}$ prediction: it might be that the $b d c$ term applied to an individual interfering signal is not the same for all pulsed interfering sources. Moreover, the blanker duration activation is not considered. The effect on $R_{I, i}$ is difficult to predict since it will depend on the analyzed interfering scenario. For example, for DME/TACAN, since the pulse arrivals follow a uniform distribution, the approximation appears to be valid. For JTIDS/ MIDS signals, the approximation is looser as seen in section V.

3) The last assumption first assumes that blanker activation by any RFI source (above-threshold pulse arrivals) follows a time uniform distribution for considering above-toabove threshold pulses collisions in addition to not consider the blanker duration activation [3]; and second neglects one type of collisions, below-to-below threshold pulses collisions. On one hand, the time uniform assumption and no blanker activation duration consideration neglect the true scenario with its true statistical collisions. Nevertheless, note that a joint $b d c$ value is proposed for DME/TACAN interference sources which take into account this type of collisions [1][9]. Therefore, final $b d c$ should have a reduced estimation precision in comparison with the value which could be estimated when considering the true (or worst) scenario. On the other hand, below-threshold pulses with below-threshold pulses collisions are not considered since each $b d c_{i}$ term is calculated only considering the $i^{\text {th }}$ interfering signal source; therefore, $b d c$ is underestimated from this perspective. The final effect on the $b d c$ computation is difficult to evaluate but a priori leans towards an underestimation.

\section{E. New proposal for $R_{I, i}$ computation using $S S C$}

A new method for the computation of $R_{I, i}$ is presented in [3]. This new proposed method addresses some of the shortcomings of DO-292 model presented in previous section II.D. More specifically, this method proposes to use the Spectral Separation Coefficient (SSC) between the useful L5/E5a GNSS signal PSD and the interfering signal true PSD (1st limitation); moreover, this method introduces new duty cycle terms and blanking duty cycle terms to model the pulse collisions impact on $R_{I, i}$ (2nd limitation). The mathematical model is given below; note that from [3], $G_{l}$, receiver antenna gain for the $i^{\text {th }}$ interfering signal, and $L_{i}^{q}$, receiver quantization losses for the $i^{\text {th }}$ interfering signal, are assumed to be included in $P_{\text {peak }, i}^{\text {out }}$.

$$
R_{I, i}=\frac{\beta_{i}\left(\Delta f_{i}\right)}{N_{0}(1-b d c) \beta_{0}} P_{\text {peak }, i}^{\text {out }} d c_{i}^{p b}\left(1-b d c^{i}\right) \operatorname{SSC}_{i}\left(\Delta f_{i}\right)
$$




$$
\begin{gathered}
\beta_{0}=\int_{-\infty}^{+\infty}\left|H_{R F}(f)\right|^{2} \bar{S}_{c_{m}}(f) d f \\
\beta_{i}\left(\Delta f_{i}\right)=\int_{-\infty}^{+\infty}\left|H_{R F}(f)\right|^{2} \bar{S}_{i, B B}\left(f-\Delta f_{i}\right) d f \\
\operatorname{SSC}_{i}\left(\Delta f_{i}\right)=\int_{-\infty}^{+\infty} \bar{S}_{i, P B f}\left(f-\Delta f_{i}\right) \bar{S}_{c_{m}}(f) d f \\
d c_{i}{ }^{p b}=d c_{i}\left(1-l d c_{i}\right)
\end{gathered}
$$

Where $\beta_{0}$ is the thermal noise power degradation due to RFFE filter and correlator, $\beta_{i}\left(\Delta f_{i}\right)$ is equivalent to the RFFE filter frequency dependent rejection (FDR) to the received RFI signal, $b d c^{i}$ is the equivalent blanking duty cycle applied to the $i^{\text {th }}$ interfering signal (not generated by itself); $\bar{S}_{i, B B}$ and $\bar{S}_{i, P B f}$ are the $i^{\text {th }}$ baseband before-blanker and post-blanker filtered normalized interfering signal PSDs; $\bar{S}_{c_{m}}$ is the local replica's normalized PSD of the $m^{\text {th }}$ PRN code; $d c_{i}$ is the post-blanker duty cycle of the $i^{\text {th }}$ interfering signal exclusive of pulse collisions; $l d c_{i}$ is the $i^{t h}$ interfering signal duty cycle loss due to blanking triggered by itself; $l d c_{i} \epsilon[0,1]$.

From equation (7), it can be observed that the loss of power introduced by the blanker is separated in two factors. First, $l d c_{l}$ represents the loss of power due to the blanker activation by the $t^{t h}$ interfering signal itself exclusive of pulse collisions. Second, $b d c^{l}$ represents the loss of power due to the activation of the blanker, which affects the $t^{\text {th }}$ interfering signal, by other interfering signals. Nevertheless, note that $b d c^{l}$ is a priori only equivalent to the percentage of time that the blanker is activated and not to the $l^{\text {th }}$ interfering signal power loss except for continuous or square pulse envelope interfering signals. Finally, from equation (7), if $\bar{S}_{i, P B f}(f)$ is assumed to have a flat spectrum on the RFFE filter and if $b d c^{i} \approx b d c$, equation (4) is found.

\section{JTIDS/MIDS DESCRIPTION}

\section{A. Systems and signal description}

The Joint Tactical Information Distribution System (JTIDS) and the Multifunctional Information Distribution System (MIDS) are military aeronautical digital tactical communication, navigation and identification systems which are operated on land, sea and airborne platforms in many countries worldwide. The JTIDS and MIDS produce the same waveform and are the radio terminals for transmission of Link 16 . The waveform is a hybrid direct sequence and frequency hopping spread-spectrum system that operates on 51 different carrier frequencies in the frequency bands of $969-1008 \mathrm{MHz}, 1053-1065 \mathrm{MHz}$ and $1113-1206 \mathrm{MHz}$ (see Fig. 4) [10]. It operates on frequency channels in the region surrounding and including the GPS/Galileo L5/E5a frequency band. A remap capability has been implemented where it would have the capability to operate on as few as 37 carrier frequencies, which could result in added pulse density within the L5/E5a band when compared to the 51 carrier case.

JTIDS/MIDS employs time division multiple access (TDMA) to accommodate multiple users in a network with 128 timeslots per second. Each time slot lasts $7.8125 \mathrm{~ms}$ with transmission message intervals of $929 \mu \mathrm{s}, 3.354 \mathrm{~ms}$ or $5.77 \mathrm{~ms}$ within assigned time slots depending on the function and the amount of information to be exchanged. The information transmission period is constituted of 72,258 or 444 pulses for the transmission periods respectively [10]. Transmissions in this period consist of a $6.4 \mu \mathrm{s}$ transmitted pulse (as measured at the $90 \%$ power level) followed by an off time of $6.6 \mu \mathrm{s}$. The frequency hops to one of the 51 carrier frequencies (or fewer down to 37) during each $13 \mu$ s interval [10]. The pattern is designed such that the use of the carriers is distributed uniformly in frequency and consecutive transmitted pulses will operate at least $30 \mathrm{MHz}$ apart. The $6.4 \mu \mathrm{s}$ pulses are formed by 32 chips of 200ns [10].

\section{B. JTIDS/MIDS Operational Conditions}

As a condition of its operation, the JTIDS transmissions must not affect the operation of other equipment in the frequency band and its waveform characteristics have been carefully chosen to promote compatibility. Levels of operations allowed national spectrum authorities of all nations permitting its operations within their territory are limited by the number of pulses or the percent time slot duty factor (TSDF) that can be transmitted in the environment within a Geographic Area (GA). 100\% Time slot duty factor (TSDF) is defined as 396288 pulses within a 12 second interval. It is usually expressed as a two-term parameter with a top number referring to the percent TSDF within a network or geographic area and a bottom parameter that reflects the TSDF of the high single user platform. It is not meant to be a ratio. For example, 100 / $50 \%$ TSDF represents 100\% TSDF in a network or geographic area and 50\% represents the TSDF of highest single user. The number of pulses or TSDF is determined from the network design.

To support the impacts analysis, different models are derived to represent the conditions in the various nations. See section III.D. For example, GA limits of $100 \%$ TSDF of all units within a $100 \mathrm{NM}$ radius and $400 \%$ within a $200 \mathrm{NM}$ radius around each platform or operating area are applied, known as Platform Centric (PC) GA. This is equivalent to the Case 8 model used in [1]. Other examples include the $100 \%$ TSDF within a 100 NM radius in a GA without a $400 \%$ TSDF within a $200 \mathrm{NM}$ radius limit. Finally, a different GA definition consists of a $100 \%$ TSDF limit within a radius of $50 \mathrm{NM}$ around Any Point in Space (APIS). Models in this analysis will accommodate these GA definitions.

\section{Signal Time-domain Waveform and Power Spectrum (PS) \\ Description for the Purpose of Interference analysis}

In this section, the time-domain JTIDS/MIDS signal as well its PS are described for interference purposes: the $C / N_{0}$ degradation caused to the useful L5/E5a GNSS/SBAS signal by the JTIDS/MIDS signal described in this section is equivalent to the $C / N_{0}$ degradation caused by the true JTIDS/MIDS signal; therefore, the described signal should not be used for any other purposes such as demodulation or positioning performance.

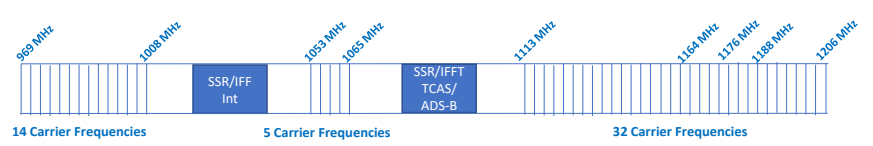

Fig. 4. JTIDS/MIDS Carrier Frequencies 


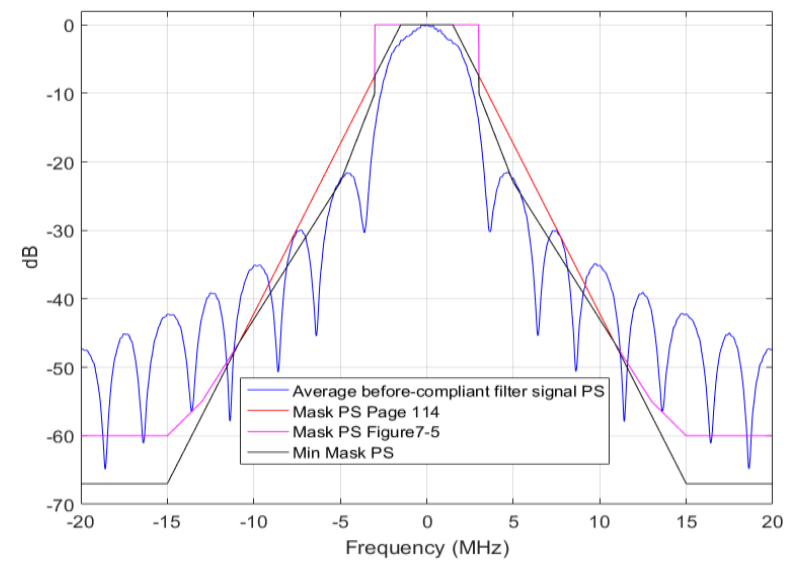

Fig. 5. $\bar{P}_{l}^{b}(f)$ PS, RTCA DO-292 [1] figure 7-4 and page 114 PS masks and minimum PS mask

More specifically, the process followed to generate the equivalent JTIDS/MIDS signal for interference analysis is described hereafter. This process consists of 3 steps. The first one consists of generating the pulse envelope. The second one introduces a Continuous-Phase Frequency-Shift Keying (CPFSK) modulation to the pulse in order to generate the pulse complex envelope or equivalent lowpass pulse. Finally, the third step involves the filtering of the PS of the previously generated pulse signal to make it compliant with the true JTIDS/MIDS pulse PS mask.

First, the normalized (maximum amplitude equal to 1) pulse envelope of the equivalent JTIDS/MIDS has a duration of $13 \mu \mathrm{s}$; the mathematical description of the pulse complex envelope, $p_{e}(t)$, is the following one: $t / 2$ for $0 \leq t \leq 0.1 ; 0.05+$ $(\sqrt{0.9}-0.05) \cdot(t-0.1) / 0.80 .05+(\sqrt{0.9}-0.05)(t-0.1) /$ 0.8 for $0.1 \leq t \leq 0.9 ; \sqrt{0.9}+(1-\sqrt{0.9}) \cdot(t-0.9) / 0.1$ for $0.9 \leq t \leq 1 ; 1$ for $1 \leq t \leq 7.2 ; 1-(1-\sqrt{0.9}) \cdot(t-7.2) / 0.1$ for $7.2 \leq t \leq 7.3 ; \sqrt{0.9}-(\sqrt{0.9}-0.05) \cdot(t-7.3) / 0.8$ for $7.3 \leq t \leq 8.1 ; 0.05-0.5 \cdot(t-8.1)$ for $8.1 \leq t \leq 8.2$ and 0 otherwise. Note that to simplify the notation, the time $t$ is expressed from now on in $\mu \mathrm{s}(1 \mu \mathrm{s} \rightarrow t=1)$.

This definition is compliant with the definition given in RTCA-DO292 [1] and in [3] where the pulse shape must be at $90 \%$ of its maximum power for $6.4 \mu \mathrm{s}$, defined as the on-time part [10]. Note that the pulse shape is different from 0 for $8.2 \mu \mathrm{s}$ as inferred by Figure 7-1 [1]. Additionally, for simulations conducted in sections IV and $\mathrm{V}$, the parts of the pulse defined in $(0 \leq \mathrm{t} \leq 0.1,0.9 \leq \mathrm{t} \leq 1,7.2 \leq \mathrm{t} \leq 7.3$ and $8.1 \leq \mathrm{t} \leq 8.2)$ are redefined using a spline interpolation in Matlab in order to have smoother transitions between the pulse main amplitude and the up and down ramps (in $0.1 \leq \mathrm{t} \leq 0.9$ and $7.3 \leq \mathrm{t} \leq 8.1$ ).

Second, a CPFSK modulation is applied to modulate the 32 chips of $200 \mathrm{~ns}$ of duration $\left(T_{c}=200 \mathrm{~ns}\right)$ carried by the $6.4 \mu \mathrm{s}$ ontime part of the pulse. The 32 chips represent a symbol of a 32Code Shift Keying mapping [10]; note that a 32 chips sequence can generate CSK symbols mapping 5 bits. The 32 chips values $\left(I_{n}, 0 \leq n \leq 31\right)$ used in each symbol are assumed to be unknown. This means that it is not possible to generate CSK symbols from true JTIDS/MIDS equipment 32 chips sequences. Therefore, in this work, a larger number of random 32 chips sequences are used to cover an average interference scenario. More specifically, 1000 random 32 chips sequences are generated and stored; then, whenever a JTIDS/MIDS signal pulse is generated, one sequence, randomly chosen among the 1000 created sequences, is used. The chosen CPSK modulation is a binary CPFSK with a peak frequency deviation, $f_{d}$, with respect to the signal central frequency of $1.4 \mathrm{MHz}$. Note that the frequency deviation is by system definition set at $1.25 \mathrm{MHz}$ [10]; nevertheless, in order to obtain a PS with a $1.7 \mathrm{MHz}$ cut-off frequency at $3 \mathrm{~dB}$, a $1.4 \mathrm{MHz}$ is required. Moreover, remember that this signal should only be applied for interference analysis and thus, this modification is acceptable. The mathematical expression of the complex envelope CPFSK modulated pulse, $p_{m}(t)$, is given in (12) [11]. The JTIDS/MIDS complex envelope pulse before PS compliance, $p_{l}^{b}(t)$, is generated by multiplying the two previous pulses as presented in (15).

$$
\begin{gathered}
p_{m}(t)=\left\{\begin{array}{cc}
1 & 0 \leq t \leq 0.9 \\
\exp \left(2 \pi f_{d} \sum_{k=0}^{n-1} I_{n}+4 \pi f_{d} T_{c} q\left(t-n T_{c}-0.9\right)\right. & 0.9 \leq t \leq 8.2 \\
0 & 8.2 \leq t
\end{array}\right. \\
n=\min (\lfloor(t-0.9) / 0.2\rfloor, 32) \\
q(t)=\left\{\begin{array}{cc}
0 & t<0 \\
t / 2 T_{c} & 0 \leq t \leq T_{c} \\
1 / 2 & T_{c}<t
\end{array}\right. \\
p_{l}^{b}(t)=p_{e}(t) p_{m}(t)
\end{gathered}
$$

Third and last, the PS of the resulting $p_{l}^{b}(t)$ must be modified to be compliant with the true JTIDS/MIDS signal PS mask. In RTCA DO-292 [1], two masks are defined in figure 74 and in the first paragraph of page 114. In order to consider the two masks as well as a true measured JTIDS/MIDS signal PS presented in figure 7-5 [1], the two masks are combined into one where the lowest value of the two is chosen for each frequency value. Note that such a choice represents the most difficult compliance from the $p_{l}^{b}(t)$ PS modification point of view. Moreover, from the measured JTIDS/MIDS signal PS in in figure 7-5 [1], it seems to better match the measured PS of page 114 PS mask than figure 7-4 mask (specially for low frequencies). Therefore, the choice made in this work seems appropriate. Fig. 5 presents the normalized PS resulting from the average of the $1000 p_{l}^{b}(t)$ pulses generated from the 1000 different CSK sequences, $\bar{P}_{l}^{b}(f)$, the PS masks defined in page 114 and figure 7-5 [1], and the resulting minimum PS mask.

In order to make $\bar{P}_{l}^{b}(f)$ compliant with the defined minimum PS mask, called $\bar{P}_{l}(f)$, the method implemented in this work consists in applying a filter directly in the frequency domain in order to avoid the filter's definition in the time domain. The filter transfer function is denoted $H_{c}(f)$ and the complex envelope compliant JTIDS/MIDS pulse equivalent for interference analysis purposes is denoted as $p_{l}(t)$.

$$
p_{l}(t)=\operatorname{IFT}\left[\operatorname{FT}\left[p_{l}^{b}(t)\right] H_{c}(f)\right]
$$

The filter must be conceived so that the compliant signal PS is always below the minimum PS mask; moreover, in this work, an additional imposed constraint on the signal PS is to have the secondary lobes smaller than $-23 \mathrm{~dB}$. Fig. 6 presents the complex envelope after-compliant filter PS of the generated equivalent for interference analysis purposes JTIDS/MIDS pulse, $\bar{P}_{l}(f)$, as 
well as the squared filter's transfer function, the generated before-compliant filter JTIDS/MIDS pulse, $\bar{P}_{l}^{b}(f)$, and the minimum PS mask.

Finally, in order to generate the different compliant JTIDS/MIDS pulses at the right carrier frequency, $f_{c}$, determined by the frequency hopping sequence of each JTIDS/MIDS user transmitter, the compliant complex envelope JTIDS/MIDS pulse, $p_{l}(t)$, is transformed into its passband expression: $p(t)=$ $\operatorname{Re}\left[p_{l}(t) e^{j 2 \pi f_{c} t}\right]$. Fig. 7 presents the before-compliant filter and after-compliant filter JTIDS/MIDS time-domain pulses. From this figure and its zoom, it can be observed that the aftercompliant-filter pulse has smoother transitions than the beforecompliant-filter one whereas the pulse envelope and the CPFSK modulation remains the about same.

\section{Interfering scenarios}

Two JTIDS/MIDS scenario models have been considered in this analysis that would include the Case 8 specified in RTCA documentation [1] and applicable models representative of other relevant GAs. These models are represented as JTIDS/MIDS point sources defined by signal power level, TSDF and RFI emitter-victim receiver distance. Note that power level is the term used in JTIDS/MIDS notation, which is equivalent to previously defined antenna output received peak power, $P_{\text {peak }, i}^{\text {out }}$.

1) Case 8: As mentioned above the Case 8 GA environment model is representative of JTIDS/MIDS usage in the US and was used for the RTCA analyses [1]. See Fig. 8: Point sources used to represent this model include a Foreground (FG) user with a received power level at the GNSS receiver antenna port of $-35 \mathrm{dBm}$ with a TSDF level of $50 \%$ at approximately 1000 feet; a Background (BG) user with a power level of $-60 \mathrm{dBm}$ and a TSDF level of $50 \%$ at approximately $5 \mathrm{NM}$; a point source R1 with a power level of $90 \mathrm{dBm}$ representing 300\% TSDF of all users between $100 \mathrm{NM}$ and 200 $\mathrm{NM}$ and a point source R2 with a power level of $-100 \mathrm{dBm}$ representing 300\% TSDF of all users beyond 200 NM. This environment was sometimes referred to as " $100 / 50 /(300)(300)$ " [1]. It should be noted that the $-90 \mathrm{dBm}$ received signal level in $\mathrm{R} 1$ was chosen as a worst case scenario level as it would result in the strongest signal level that would not be blanked by the receiver pulse blanker. Additionally, if a case with the blanker threshold set to $-91 \mathrm{dBm}$ is analyzed, the $-90 \mathrm{dBm}$ point sources were changed to $-91 \mathrm{dBm}$ in order to maintain the same level of conservatism.

2) 50 NM APIS: Environment model is shown in Fig. 9 and characteristics are summarized in TABLE I. In this model, FG and BG are the same as in case 8 scenario. Moreover, point source R1 with a power level of $90 \mathrm{dBm}$ representing $600 \%$ TSDF at approximately $102 \mathrm{NM}$, a point source $\mathrm{R} 2$ with a power level of $99 \mathrm{dBm}$ representing 600\% TSDF at approximately $176 \mathrm{NM}$ and a point source R3 with a power level of $101 \mathrm{dBm}$ representing 600\% TSDF at approximately 204NM.

3) Case $8 A$ : For the scenario where there is no a TSDF management ring between $100 \mathrm{NM}$ and $200 \mathrm{NM}$ that is specified in Case 8, a modified set of point sources is utilized. See Fig. 10. In this model, FG and BG are the same as in case 8 scenario. Moreover, the TSDF of point source R1 with a power level of $90 \mathrm{dBm}$ is set to $600 \%$ and no point source $\mathrm{R} 2$ is considered.

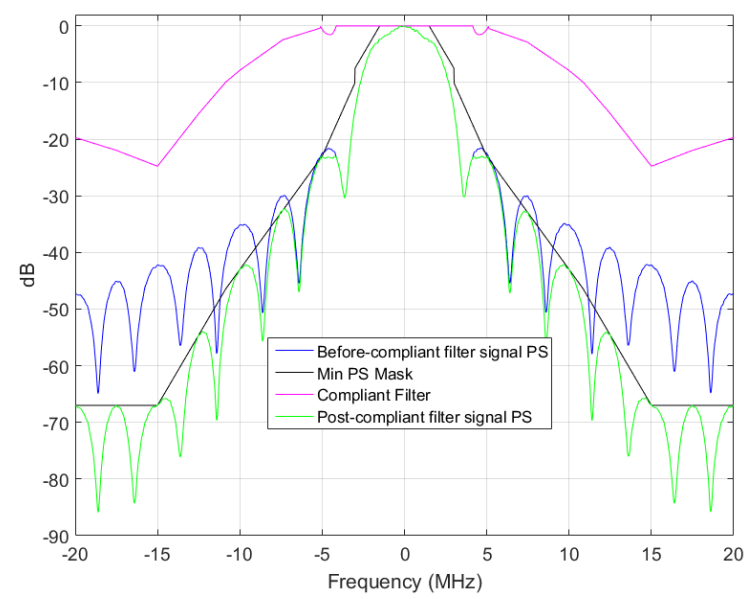

Fig. 6. Compliant signal PS with minimum PS mask
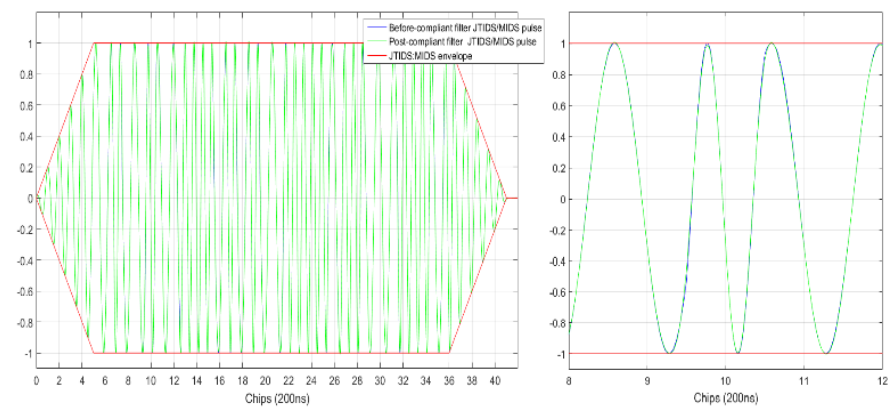

Fig. 7. Generated JTIDS/MIDS time-domain pulse

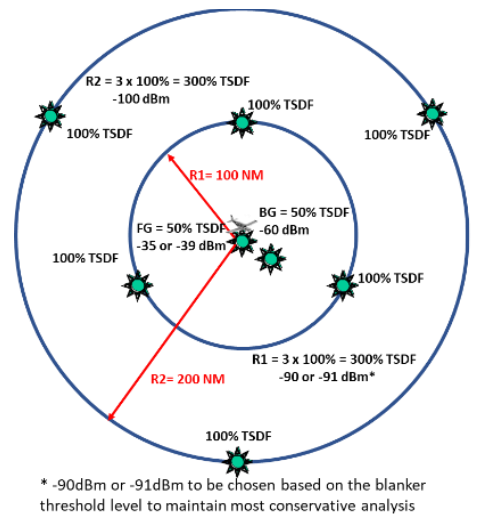

Fig. 8. JTIDS/MIDS Case 8 Environment

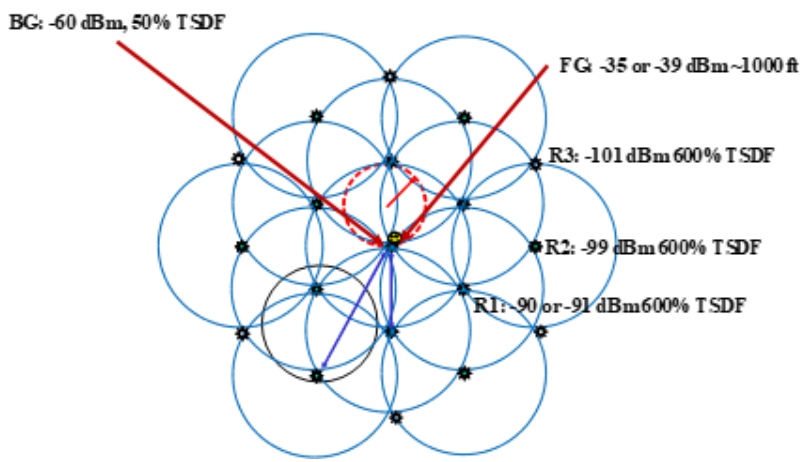

Fig. 9. JTIDS/MIDS 50 NM Any Point in Space Case Environment 


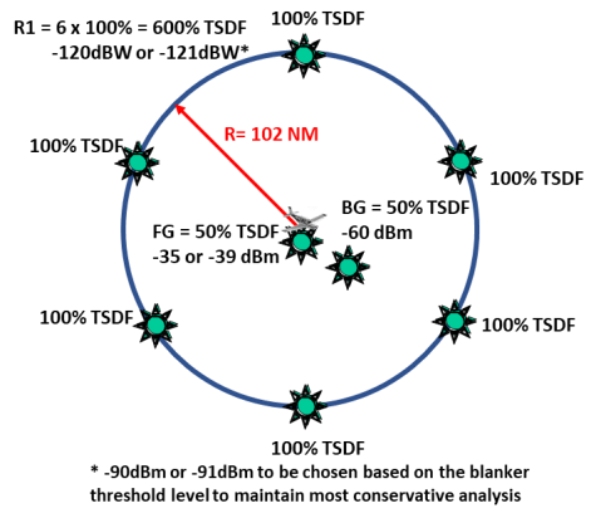

Fig. 10. JTIDS/MIDS Case 8A Environment

TABLE I. JTIDS/MIDS 50 NM ANY POINT IN SPACE CASE ENVIRONMENT SUMMARY

\begin{tabular}{|c|c|c|c|c|c|}
\hline Point & $\boldsymbol{F} \boldsymbol{G}$ & $\boldsymbol{B G}$ & $\boldsymbol{R} \boldsymbol{1}$ & $\boldsymbol{R} 2$ & $\boldsymbol{R 3}$ \\
\hline TSDF Level (\%) & 50 & 50 & 600 & 600 & 600 \\
\hline Peak Power (dBm) & -35 & -60 & -90 & -99 & -101 \\
\hline Distance (NM) & $\sim 1000$ feet & 5 & 102 & 176 & 204 \\
\hline
\end{tabular}

\section{IV. $C / N_{0}$ DEGRADATION VALIDATION RESUlTS FOR SIMPLIFIED} JTIDS/MIDS SIGNALS AND SCENARIO

In comparison to the general RTCA DO-292 $C / N_{0}$ and $R_{I, i}$ expressions presented in section II.C which present 3 limitations, the new formulas proposed in section II.E and in [3], provide a more accurate $R_{I, i}$, and $C / N_{O}$ mathematical modelling since they address the first two limitations. However, the verification of these new proposed formulas for JTIDS/MIDS signals is not yet done. The verifications of the $R_{I, i}$ formula presented in section II.E and the general $C / N_{0}$ degradation formula presented in section II.C are conducted in this section.

The main challenge in conducting this verification lies in the fact that the exact calculation of the post-blanker PSD and $b d c^{i}$ of each individual interfering JTIDS/MIDS signal $i$ is cumbersome to make and varies from interfering scenario to interfering scenario: $b d c^{i}$ is different between case 8 , case $8 \mathrm{~A}$ and 50 NM APIS. Therefore, the verification conducted in this section will be made in simplified environments and with simplified signals in order to remove the collisions events as well as to have a high-accurate estimate of the post-blanker PSD signal, $\bar{S}_{i, P B f}$. Note that the verifications are performed by comparing the $C / N_{0}$ degradation results predicted with the new proposed $R_{I, i}$ formula with respect to simulation results obtained when using the compliant JTIDS/MIDS signal of section III.C.

\section{A. Signal and Scenario description}

The selected scenario is derived from the case 8 interfering scenario. However, in order to avoid collisions events, only one type of user (FG, BG, etc.) and only one user with an associated $T S D F_{i}$ will appear for any predicted/simulated result; therefore, $b d c^{i}$ and $\bar{S}_{i, P B f}$ are easily known and $b d c=b d c_{i}, R_{I}=R_{I, i}$. A $T S D F_{i}$ value equal to $50 \%$ is considered for each type of user and each user transmits 258 pulses per slot of $7.8125 \mathrm{~ms}, T_{\text {slot }}$. The blanker threshold is set to $-90 \mathrm{dBm}$.

Moreover, for the first verification, only one JTIDS/MIDS carrier frequency centered at L5 band (1176 MHz) will be considered, meaning that the simplified JTIDS/MIDS signal is no longer a frequency hopping signal. The reason for suppressing the frequency hopping characteristic is to have pulses always triggering the blanker for user types FG and BG, and thus having a negligible $R_{I}$ contribution. Note that if the frequency hopping characteristic is not disabled, FG and BG users can also generate non-negligible $R_{I}$ values for carrier frequencies outside the RFFE bandwidth. For the second verification, the frequency hopping will be allowed.

\section{B. Predicted results analytical derivation}

To predict the $C / N_{0}$ degradation results, the $b d c_{i}$ and $R_{I, i}$ values of each user type must be calculated; where index $i$ determines the user type. Two types of analytical derivations will be made depending on whether the frequency hopping is activated or not. In fact, note that one difficulty in analyzing JTIDS/MIDS signals with respect to other RFI is the frequency hopping property; thus, when customizing equations (7) to (11), the carrier frequency variation influence must be added; from now on, index $j$ identifies the pulse carrier frequency.

The first analytical derivation will address the case where the frequency hopping is deactivated but which is still dependent on the pulse carrier frequency. For this case, the calculation of $R_{I, i}$ is reduced to the calculation of $b d c^{i}, d c_{i}, l d c_{i}, \beta_{i}$ and the $S C C_{i}$ term. $\beta_{i}\left(\Delta f_{j}\right)$ and $S C C_{i}\left(\Delta f_{j}\right)$ calculations are commented later; moreover, since only one user is considered, $b d c^{i}$ is always 0 .

Concerning $d c_{i}$, the same calculation developed in [3] can be made: the RFI signal waveform power of the $i^{\text {th }}$ JTIDS/MIDS user type at the RFFE input, $P_{w f, i}$, is equal to $P_{\text {peak }, i}^{\text {out }} \cdot d c_{i}$ (since for all frequency carriers, the transmitted JTIDS/MIDS pulse power is the same); and the power of a signal can be calculated as the energy divided by time, $P_{w f, i}=E / T$. The signal energy can be calculated as the area below the instantaneous energy $|s(t)|^{2}$ [11]. The energy of 1 pulse can be calculated by integrating $|p(t)|^{2}$, and the result yields, $E_{1 p}=P_{\text {peak }, i}^{\text {out }} \cdot T_{\text {eq }} / 2$ where $T_{e q}=7.2 \mu s$. Then, since 258 pulses are transmitted per slot and since only $T S D F_{i}$ slots transmit pulses, in average inside a period of $T_{\text {slot }}\left(T_{\text {slot }}=7812.5 \mu \mathrm{s}\right)$, the total energy is equal to $E=258 \cdot T S D F \cdot E_{1 p u l s e}$. Thus, $d c_{i}$ is equal to $129 \cdot T S D F$. $T_{e q} / T_{\text {slot }}$ (equal to half the value in DO-292 [1]). Moreover, note that $d c_{i}$ is defined irrespective of the carrier frequency $j$.

Concerning $l d c_{i}$, with $l d c_{i}=l d c_{i}\left(\Delta f_{j}\right)$ when frequency hopping is not allowed, $\left(l d c_{i}\left(\Delta f_{j}\right)\right.$ is $l d c_{i}$ for pulse at frequency carrier $j$ ) the calculation is quite straightforward. If the pulse does not trigger the blanker, $l d c_{i}=0$ since there are no other interfering sources; if the blanker threshold is exceeded, for the specific scenarios and chosen signals, $l d c_{i}$ can be approximated to 1 . Expressing $R P_{i}^{j}$ as the ratio between the blanker threshold, $T h$, and the pulse peak power of the JTIDS/MIDS user $i$ transmitted at frequency carrier $j$ at the RFFE output, $P_{\text {peak,i,f }}^{j}$, the pulse activates the blanker when $R P_{i}^{j} \leq 1$.

$$
R P_{i}^{j}=T h / P_{\text {peak,i,f }}^{j}
$$

Finally, concerning $b d c_{i}$, with $b d c_{i}=b d c_{i}\left(\Delta f_{j}\right)$ when frequency hopping is not allowed, $\left(b d c_{i}\left(\Delta f_{j}\right)\right.$ is $b d c_{i}$ for pulse 
at frequency carrier $j$ ), the calculation is also quite straightforward. If the pulse does not trigger the blanker, $b d c_{i}=$ 0 . If the pulse triggers the blanker threshold, for the considered peak power values of FG and RG1, it can be assumed that the blanker threshold is triggered during all the $8.2 \mu \mathrm{s}$ pulse duration. Note that as assumed in RTCA DO292 [1] analyses, $8.4 \mu \mathrm{s}$ is used in order to include "pulse stretching" caused by the blanking circuitry. Thus, $b d c_{i}$ is calculated by dividing the total blanking time in one time slot by the time slot duration and considering that only TSDF slots contain pulses (see equation (18) for $B$ containing only one frequency carrier, $N_{c}=1$ ).

The second analytical derivation will address the case where the frequency hopping is activated. With respect to the previous case, the calculations of $b d c_{i}$ and $R_{I, i}$ must be modified since the JTIDS/MIDS pulses central frequency, $f_{c_{j}}$, varies from pulse to pulse as well as $\Delta f_{j}=f_{c_{j}}-f_{L 5}$. Modelling the JTIDS/MIDS pulse central frequency as a random variable with equiprobable outputs (each available JTIDS/MIDS carriers/channels is assumed to be used on average the same number of times), $b d c_{i}$ is calculated as:

$$
b d c_{i}=E_{\Delta f_{j}}\left[b d c_{i}\left(\Delta f_{j}\right)\right]=\frac{T S D F}{N_{c}} \sum_{\Delta f_{j} \in B} 258 \frac{g\left(T h, \Delta f_{j}\right)}{T_{\text {slot }}}
$$

Where $T h$ is the blanker threshold; $N_{c}$ is the number of different JTIDS/MIDS carriers/channels (either 37 or 51 carriers); $B$ is the ensemble of all possible $\Delta f_{j}$ values; $f_{L 5}-f_{c_{j}}$; $g$ is a function determining the JTIDS/MIDS pulse duration (expressed in $\mu s$ ) which is blanked for a given $T h$ value and the frequency difference $\Delta f_{j}$ between L5/E5a and the JTIDS/MIDS carrier frequency:

$$
g\left(R P_{i}^{j}, \Delta f_{j}\right) \approx\left\{\begin{array}{cl}
0 & 1 \leq R P_{i}^{j} \\
6.2+2 \cdot\left(1-R P_{i}^{j}\right) & 1>R P_{i}^{j}
\end{array}\right.
$$

Finally, $R_{I, i}$ can be calculated as $\left(b d c^{i}=0\right)$ :

$$
\begin{gathered}
R_{I, i}=E_{\Delta f_{j}}\left[R_{I, i}^{\Delta f_{j}}\right]=\frac{P_{\text {peak }, i}^{\text {out }} d c_{i}}{2 N_{0}(1-b d c) \beta_{0}} . \\
\sum_{\Delta f_{j} \in B}\left(1-l d c_{i}\left(R P_{i}^{j}, \Delta_{f_{j}}\right)\right) \beta_{j}\left(\Delta_{f_{j}}\right) S S C\left(\Delta f_{j}\right) \\
l d c_{i}\left(R P_{i}^{j}, \Delta f_{j}\right) \approx\left\{\begin{array}{cc}
0 & 1 \leq R P_{i}^{j} \\
\left(6.2+\left(1-\left(R P_{i}^{j}\right)^{2}\right) / 7.2\right. & 1>R P_{i}^{j}
\end{array}\right.
\end{gathered}
$$

Finally, to calculate $\beta_{i}\left(\Delta f_{j}\right)$ and the $S C C_{i}\left(\Delta f_{j}\right.$, in both cases, frequency hopping activated or not, $\bar{S}_{i, B B}(f)$ is equal to the postcompliant filter PSD of Fig. 6, and $\bar{S}_{i, P B f}(f)$ is approximated as $\bar{S}_{i, B B}(f) \cdot\left|H_{R F}(f)\right|^{2}$. Note that this approximation is only accurate for not blanked pulses (no pulse collisions); but, for the analyzed scenarios $\left(1 \gg R P_{i}^{j}\right.$ ), the contribution to $R_{I}$ of the not blanked parts of the blanked pulses is negligible.

\section{Comparison between predicted and simulation results}

TABLE II. presents the intermediate predicted, $d c_{i}, l d c_{i}$ and $b d c$ values, and the predicted $C / N_{0}$ degradation (calculated from equation (3) with $I_{0, W B}=0$ ) for the case where the
JTIDS/MIDS frequency hopping is not activated. Moreover, TABLE II. also presents the results obtained by simulating the different simplified scenarios and characteristics. TABLE III. presents the same results as TABLE II. for the case where the JTIDS/MIDS frequency hopping is activated. The calculation of filtered peak power values, $P_{\text {peak }, i, f}^{j}$ is conducted as $P_{\text {peak }, i}^{\text {out }}$. $\beta_{i}\left(\Delta f_{j}\right)$; note that this expression is an approximation for signals falling in the RF filter transition band.

The simulated signal is constructed by using the compliant JTIDS/MIDS pulses presented in section III.C and by using the slot structure presented in section III.A for 258 pulses. In this regard, the initial random jitter value at the beginning of each slot is generated from a uniform random distribution $[0,1.9 \mathrm{~ms}]$. Note that the maximum value is chosen to cover a propagation range of $300 \mathrm{NM}$. Finally, the simulated $C / N_{0}$ degradation is computed by first generating a 6-seconds L5/E5a PRN code-like signal plus the simplified JTIDS/MIDS signal and by second applying a simplified GNSS software receiver with a $C / N_{0}$ estimator, $C / N_{0}=E\left[I^{2}\right] / \operatorname{var}[Q]$ (from $2 \mathrm{~s}$ to $6 \mathrm{~s}$ ). The blanker threshold is set to $-90 \mathrm{dBm}$ and $N_{0}$ is set to $-200 \mathrm{~dB}-\mathrm{Hz}$.

From TABLE II. and TABLE III. , it can be observed that the predicted values match very closely the predicted results since the $C / N_{O}$ degradation deviation is always below $0.13 \mathrm{~dB}$. Note that this deviation also includes the uncertainty of the $C / N_{O}$ estimator and that $R_{I}$ simulation results are obtained isolating $R_{I}$ in equation (3). Therefore, the $R_{I}$ formula proposed in [3] is also validated for JTIDS/MIDS signals.

\section{V. $C / N_{0}$ DEGRADATION RESUlTS FOR JTIDS/MIDS SIGNALS SCENARIO}

In this section, several JTIDS/MIDS $C / N_{0}$ degradation results are predicted for case 8 and 50NM APIS interfering scenarios and they are compared to simulated results. Moreover, the impact of some receiver parameter values, RFFE bandwidth and blanker threshold, on the $C / N_{O}$ degradation is analyzed to provide recommendations.

TABLE II. $\quad C / N_{0}$ DEGRADATION COMPARISON BETWEEN PREDICTED AND SIMULATED RESULTS FOR SIMPLIFIED JTIDS/MIDS SIGNAL WITHOUT FH

\begin{tabular}{|l|c|c|c|c|}
\hline \multicolumn{1}{|c|}{ User } & $\boldsymbol{F G}$ & $\boldsymbol{B G}$ & $\boldsymbol{R G 1}$ & $\boldsymbol{R G}$ \\
\hline $\boldsymbol{T S D F}(\%)$ & 50 & 50 & 50 & 50 \\
\hline ldc $_{\boldsymbol{i}}$ & 1 & 1 & 0 & 0 \\
\hline Predicted $\boldsymbol{b d c}$ & 0.1354 & 0.1354 & 0 & 0 \\
\hline Simulated $\boldsymbol{b d} \boldsymbol{c}$ & 0.1349 & 0.1328 & 0 & 0 \\
\hline Predicted $\boldsymbol{R}_{\boldsymbol{I}}$ & $\sim 0$ & $\sim 0$ & 0.6089 & 0.06089 \\
\hline Simulated $\boldsymbol{R}_{\boldsymbol{I}}{ }^{*}$ & $\sim 0$ & $\sim 0$ & 0.5596 & 0.0568 \\
\hline Pred Deg $\boldsymbol{C} / \boldsymbol{N}_{\boldsymbol{o}}$ & 0.63 & 0.63 & 2.07 & 0.26 \\
\hline Sim Deg $\boldsymbol{C} / \boldsymbol{N}_{\boldsymbol{0}}$ & 0.66 & 0.56 & 1.93 & 0.24 \\
\hline
\end{tabular}

TABLE III. $C / N_{0}$ DEGRADATION COMPARISON BETWEEN PREDICTED AND SIMULATED RESULTS FOR SIMPLIFIED JTIDS/MIDS SIGNAL WITH FH

\begin{tabular}{|l|c|c|c|c|}
\hline \multicolumn{1}{|c|}{ User } & $\boldsymbol{F G}$ & $\boldsymbol{B G}$ & $\boldsymbol{R G I}$ & $\boldsymbol{R G 2}$ \\
\hline $\boldsymbol{T S D F}(\%)$ & 50 & 50 & 50 & 50 \\
\hline Predicted $\boldsymbol{b d c}$ & 0.0549 & 0.403 & 0 & 0 \\
\hline Simulated $\boldsymbol{b} \boldsymbol{d} \boldsymbol{c}$ & 0.0537 & 0.0416 & 0 & 0 \\
\hline Predicted $\boldsymbol{R}_{\boldsymbol{I}}$ & $4.22 \mathrm{e}-4$ & $2.37 \mathrm{e}-4$ & 0.0536 & 0.00536 \\
\hline Simulated $\boldsymbol{R}_{\boldsymbol{I}}$ * & 0.0187 & $\sim 0$ & 0.0495 & 0.0062 \\
\hline Pred $\boldsymbol{D e g} \boldsymbol{C} / \boldsymbol{N}_{\boldsymbol{o}}$ & 0.25 & 0.18 & 0.23 & 0.023 \\
\hline Sim $\boldsymbol{D e g} \boldsymbol{C} / \boldsymbol{N}_{\boldsymbol{0}}$ & 0.32 & 0.17 & 0.21 & 0.027 \\
\hline \multicolumn{5}{|r}{ * Simulated $R_{\boldsymbol{I}}$ is calculated from equation (3) }
\end{tabular}


Simulated $C / N_{0}$ degradation is computed by generating first a 6-seconds L5/E5a PRN code-like signal plus the JTIDS/MIDS signals corresponding to the analyzed scenario and noise. Second, a simplified GNSS software receiver with a $C / N_{0}$ estimator is applied (from $2 \mathrm{~s}$ to $6 \mathrm{~s}$ ). JTIDS/MIDS signals are generated following the time-domain signal description given in section III.C. Finally, it is assumed that all the JTIDS/MIDS users are synchronized: they share the same time reference, the time slots are synchronized and thus, the victim receiver sees the beginning of each time slot from a different user as a function of the propagation delay (distance between JTIDS/MIDS user and victim) plus the time-slot initial random jitter (uniform random distribution $[0,1.9 \mathrm{~ms}])$.

TABLE IV. compares RTCA DO292 [1] results and predicted results using [1] formula, [1] formula with updated $d c_{i}$ (new value is half the original one as derived in section IV.B) and proposed formula (section II.E). RF filter bandwidth is $20 \mathrm{MHz}$, blanker threshold is $-90 \mathrm{dBm}$, pulse duration is $8.4 \mu \mathrm{s}$ and FG peak power is $-34.5 \mathrm{dBm}$. From this table it can be observed that $C / N_{0}$ degradation formula provided in DO-292 does not allow to obtain the published results even with the 2 factor on $d c_{i}$. Finally, proposed formula predicts a slightly $C / N_{0}$ degradation improvement with respect to [1] predicted results.

TABLE V. presents the $C / N_{0}$ degradation results, as well as $b d c$, for the case 8 interfering scenario for 37 carrier frequencies. The predictions and simulation results are given for two threshold values, $-90 \mathrm{dBm}$ and $-91 \mathrm{dBm}$, and for two different RFFE filter bandwidth, $12 \mathrm{MHz}$ and $20 \mathrm{MHz}$. The $12 \mathrm{MHz}$ filter used in this work has been derived from the $20 \mathrm{MHz}$ filter, see Fig. 2, by just reducing the useful band to 6MHZ (one-sided) and keeping the transition band's slopes. A good match between the predicted and simulated results is observed from TABLE V. The potential difference between the predicted results and the simulated results comes from the apparition of collisions which introduce uncertainties in the filtered post-blanker signal PSD and on $b d c^{i}$. For example, in simulated scenario $B W=20 \mathrm{MHz}$ and $T h=-90 \mathrm{dBm}, \mathrm{RG} 1 b d c^{i}$ is about 0.18 and RG2 $b d c^{i}$ is about 0.14 . These values are larger than the overall $b d c$ and thus the overestimation of $R_{I}$ with respect to $b d c^{i}$ is expected. However, since the predicted $C / N_{O}$ degradation is higher than the simulated one, the previous overestimation is a priori compensated by the underestimation made by using the before-blanker signal PSD instead of the true filtered post-blanker signal PSD: filtered post-blanker signal PSD is more spread and thus, in average, below-threshold pulses have a higher impact on $R_{I}$.

TABLE VI. presents the $C / N_{0}$ degradation results, as well as $b d c$, for the 50NM APIS interfering scenario for 37 carrier frequencies. The predictions and simulation results are given for the same threshold and RFFE filter bandwidth values as TABLE V. From TABLE VI. , it can also be seen a good match between predicted and simulated results, with a slightly higher difference due to the larger number of users' TSDF (RG1 TSDF from $300 \%$ to $600 \%$, RG2 TSDF from $300 \%$ to $600 \%$, and new RG2 TSDF $600 \%$ ) which further highlight the approximations made during the predicted results. Moreover, from these results it can be seen that 50NM APIS interfering scenario introduces a higher $C / N_{0}$ degradation than case $8 \mathrm{GA}$.
TABLE IV. C/N DEGRADATION COMPARISON BETWEEN [1] RESULTS, FORMULA AND PREDICTED RESULTS USING ALTERNATIVE EXPRESSIONS

\begin{tabular}{|l|c|c|c|c|}
\hline & {$[1]$ results } & [1] formula & $\begin{array}{c}\text { [1] formula } \\
\text { with new } \boldsymbol{d} \boldsymbol{c}_{\boldsymbol{i}}\end{array}$ & $\begin{array}{c}\text { Proposed } \\
\text { Method }\end{array}$ \\
\hline $\boldsymbol{B d} \boldsymbol{c}$ & 0.101 & 0.0951 & 0.0951 & 0.0951 \\
\hline $\boldsymbol{R} \boldsymbol{i}$ & 0.377 & 0.8375 & 0.4197 & 0.3545 \\
\hline $\boldsymbol{C} / \boldsymbol{N}_{\boldsymbol{0}} \mathrm{Deg}$ & 1.85 & 3.08 & 1.96 & 1.75 \\
\hline
\end{tabular}

TABLE V. $C / N_{0}$ DEGRADATION PREDICTIONS AND SIMULATION RESULTS FOR CASE 8 SCENARIO

\begin{tabular}{|l|c|c|c|c|}
\hline Bandwidth & \multicolumn{2}{|c|}{$\mathbf{2 0} \mathbf{M H z}$} & \multicolumn{2}{c|}{$\mathbf{1 2 ~} \mathbf{M H z}$} \\
\hline Threshold & $\mathbf{- 9 0 d B m}$ & $\mathbf{- 9 1 d B m}$ & $\mathbf{- 9 0 d B m}$ & $\mathbf{- 9 1 d B m}$ \\
\hline Sim $\boldsymbol{b d c}$ & 0.0956 & 0.0959 & 0.0772 & 0.0774 \\
\hline Pred $\boldsymbol{b d c}$ & 0.0929 & 0.0929 & 0.0808 & 0.0808 \\
\hline $\begin{array}{l}\text { Simulated } \\
\boldsymbol{C} / \boldsymbol{N}_{\boldsymbol{0}} \text { deg }\end{array}$ & $1.68 \mathrm{~dB}$ & $1.49 \mathrm{~dB}$ & $1.58 \mathrm{~dB}$ & $1.39 \mathrm{~dB}$ \\
\hline $\begin{array}{l}\text { Predicted } \\
\boldsymbol{C} / \boldsymbol{N}_{\boldsymbol{0}} \text { deg }\end{array}$ & $1.74 \mathrm{~dB}$ & $1.52 \mathrm{~dB}$ & $1.64 \mathrm{~dB}$ & $1.43 \mathrm{~dB}$ \\
\hline
\end{tabular}

TABLE VI. C/N $N_{0}$ DEGRADATION PREDICTIONS AND SIMULATION RESULTS FOR 50NM APIS SCENARIO

\begin{tabular}{|l|c|c|c|c|}
\hline Bandwidth & \multicolumn{2}{|c|}{$\mathbf{2 0} \mathbf{M H z}$} & \multicolumn{2}{c|}{$\mathbf{1 2} \mathbf{M H z}$} \\
\hline Threshold & $\mathbf{- 9 0 d B m}$ & $\mathbf{- 9 1 d B m}$ & $\mathbf{- 9 0 d B m}$ & $\mathbf{- 9 1 d B m}$ \\
\hline Sim $\boldsymbol{b d c}$ & 0.0953 & 0.0953 & 0.0772 & 0.0774 \\
\hline Pred $\boldsymbol{b d c}$ & 0.0951 & 0.0951 & 0.0808 & 0.0808 \\
\hline $\begin{array}{l}\text { Simulated } \\
\boldsymbol{C} / \boldsymbol{N}_{\boldsymbol{0}} \text { deg }\end{array}$ & $2.72 \mathrm{~dB}$ & $2.43 \mathrm{~dB}$ & $2.63 \mathrm{~dB}$ & $2.35 \mathrm{~dB}$ \\
\hline $\begin{array}{l}\text { Predicted } \\
\boldsymbol{C} / \boldsymbol{N}_{\boldsymbol{0}} \text { deg }\end{array}$ & $2.93 \mathrm{~dB}$ & $2.59 \mathrm{~dB}$ & $2.78 \mathrm{~dB}$ & $2.46 \mathrm{~dB}$ \\
\hline
\end{tabular}

From TABLE V. and TABLE VI. , it can be observed that a narrower RFFE filter bandwidth, $B W$, is beneficial for mitigating the $C / N_{0}$ degradation due to JTIDS/MIDS signals since a lower number of interfering signals are allowed inside the useful RFFE filter bandwidth. An improvement about 0.1 to $0.15 \mathrm{dBs}$ is expected. The same conclusions can be extracted by the reduction of the blanker threshold with an even better improvement of $0.3 \sim 0.4 \mathrm{dBs}$ for the 50NM APIS scenario. In fact, note that by the interfering scenario description, a threshold equal to $-91 \mathrm{dBm}$ implies a RG1 user received with a peak power equal to $-91 \mathrm{dBm}$ with respect to the $-90 \mathrm{dBm}$ received peak power for the $-90 \mathrm{dBm}$ threshold scenario. Therefore, in both cases, $b d c$ remains the same but RG1 have a peak power $1 \mathrm{~dB}$ lower and thus, a lower $C / N_{0}$ degradation is expected. Note that if RG1 users' peak power is held to $-90 \mathrm{dBm}$ for the $-91 \mathrm{dBm}$ threshold case, RG1 users' pulses inside the useful RFFE filter bandwidth will be blanked and the $C / N_{0}$ degradation improvement will be even larger. Therefore, this peak power change represents a worst-case scenario.

\section{THE MITRE CORPORATION APPROACH FOR $C / N_{0}$ DEGRADATION CALCULATIONS}

The MITRE approach consists of two different parts. One for determining $b d c$ and another for determining $R_{I}$. The former part involves consideration of the GNSS receiver RF front end (RFFE) filter effect on the JTIDS/MIDS signals from each of the carriers to determine the signal level present at the pulse blanker and the contribution to the $b d c$. The latter part examines the post blanker low level JTIDS/MIDS signals and how they contribute to the $R_{I}$ value post correlator. 


\section{A. bdc calculation}

To evaluate the JTIDS/MIDS signal levels into the receiver pulse blanker and to calculate $b d c$, equations (22) to (24) [1] are used. First, a frequency dependent rejection (FDR) calculation between the pulse spectrum of each of the JTIDS/MIDS carrier frequencies with the GNSS receiver RFFE filter selectivity is performed to determine the FDR coefficient values for those carriers. Second, these FDR coefficient values divide the peak power level for each of the modelled JTIDS/MIDS transmission sources (as in equation (23)) and the results is compared to the pulse blanker threshold, $-90 \mathrm{dBm}$ or $-91 \mathrm{dBm}$, to determine which JTIDS/MIDS pulse are blanked.

$$
\begin{aligned}
F D R\left(\Delta f_{j}\right) & =\frac{\int_{-\infty}^{+\infty} S(f) R(f) d f}{\int_{-\infty}^{+\infty} S(f) R\left(f+\Delta f_{j}\right) d f} \\
P_{\text {peak }, i, f}^{j} & =P_{\text {peak }, i}^{\text {out }} / F D R\left(\Delta f_{i}\right)
\end{aligned}
$$

Where $S(f)$ is the JTIDS/MIDS pulse spectrum (see Fig. 11), $R(f)$ is the GNSS RFFE filter response (see Fig. 2), $\Delta f_{i}$ is the JTIDS/MIDS frequency offset from (L5/E5a), $\Delta f_{i}=f_{c_{i}}-$ $f_{L 5}, P_{\text {peak }, i}^{\text {out }}$ is the JTIDS/MIDS pulse peak power at the antenna output and $P_{\text {peak,if }}$ is the JTIDS/MIDS pulse peak power at the blanker input.

Therefore, based on the number of JTIDS/MIDS carriers, $x_{i}$, that produce a signal level stronger than the blanker level $\left(P_{\text {peak }, i, f}^{j}>T h\right)$ and the TSDF for each of the modelled sources, the total number of pulses exceeding the threshold for the respective point source $i$ is used to calculate $b d c_{i}$ for that point source $i$. See the calculation shown in equation (24).

$$
b d c_{i}=\frac{T S D F_{i} \cdot 8.4 \cdot 258 \cdot x_{i}}{7812.5 \cdot N_{c}}
$$

Where $N_{c}$ is the number of Pseudo-randomly selected carriers (either 37 or 51). Note that equation (24) is equivalent to equation (18) when $R P_{i}^{j} \ll 1$ (where 8.2 has been converted to 8.4 in order to take into account pulse stretching due to blanking circuitry [1]). Fig. 12 provides the FDR coefficient value results of the FDR calculation that are used to derive the $b d c$ given the peak of the received JTIDS/MIDS signal level and the TSDF of the different JTIDS/MIDS modelled signal sources. Given the received signal peak power levels from the foreground and background terminals of $-35 \mathrm{dBm}$ and $-60 \mathrm{dBm}$ maximum, the following $b d c$ values are found with the blanker values of $-90 \mathrm{dBm}$ and $-91 \mathrm{dBm}$ respectively with 51 or 37 carrier frequencies. See TABLE VII. . Note that the point sources R1, $\mathrm{R} 2$ and R3 will not contribute to the $b d c$ as the received levels are too weak; therefore, case 8, case 8A and 50NM APIS have the same $b d c$ value as a function of the number of randomly selected carriers, 37 or 51. Finally, the total $b d c$ for the JTIDS/MIDS case would be the addition of the $b d c_{i}$ of all the individual point sources (FG, BG, R1, etc.).

\section{B. $R_{I}$ calculation}

Equation (25) was used to evaluate the low level JTIDS/MIDS post receiver blanker signal levels into the receiver correlator in order to calculate the factor of $R_{I, i}$ for the different point sources modelled in section III.D. Total $R_{I}$ is calculated by adding the different $R_{I, i}$ values (equation (2)).

$$
\begin{array}{r}
R_{I, i}=\frac{1}{N_{0} \cdot B W} \sum_{\Delta f_{j} \in B} P_{\text {peak }, i, f}^{j} \cdot \operatorname{SSC}\left(\Delta f_{j}\right) \cdot d c_{i, j} \\
d c_{i, j}=\left\{\begin{array}{cc}
T S D F_{i} \cdot \frac{7.2 \cdot 258}{7812.5 \cdot N_{c}} & P_{\text {peak }, i, f}^{j}<T h \\
0 & P_{\text {peak }, i, f}^{j}>T h
\end{array}\right.
\end{array}
$$

Where $\operatorname{SSC}\left(\Delta f_{j}\right)$ is the Spectrum Separation Coefficient (SSC) for $j^{\text {th }}$ JTIDS/MIDS Carrier, $B$ is the ensemble of all possible $\Delta f_{j}$ values, $B W$ is the RFFE plus antenna filter bandwidth equal to $20 \mathrm{MHz}$ and $N_{0}$ is the Noise Power Density level set to $-200 \mathrm{dBW} / \mathrm{Hz}$. $d c_{i, j}$ is the JTIDS/MIDS pulse duty cycle transmitted at the carrier frequency $j$ of the JTIDS/MIDS user type $i$, where $T h$ is the blanker threshold.

For $R_{I, i}$ calculation, the Spectrum Separation Coefficients $\operatorname{SSC}\left(\Delta f_{j}\right)$ values are determined using equation (22) as for the $b d c$ calculation. This is essentially a calculation of the receiver correlator FDR of the post blanker JTIDS/MIDS pulse spectrum. Therefore, in this case, $R(f)$ represents the GNSS correlator filter response. Within equation (22), $S(f)$ is represented by the post blanker JTIDS/MIDS pulse spectrum which is shown in Fig. 11.

The GNSS receiver PN spreading code, which is similar to a $\operatorname{sinc}^{2}$ function is modelled in Fig. 13. This is used to represent the correlator response $R(f)$. The calculated SSCs from the FDR analysis is shown in Fig. 14. These SSCs are used to assess the $R_{I, i}$ based on the JTIDS/MIDS low level signals and the TSDF.

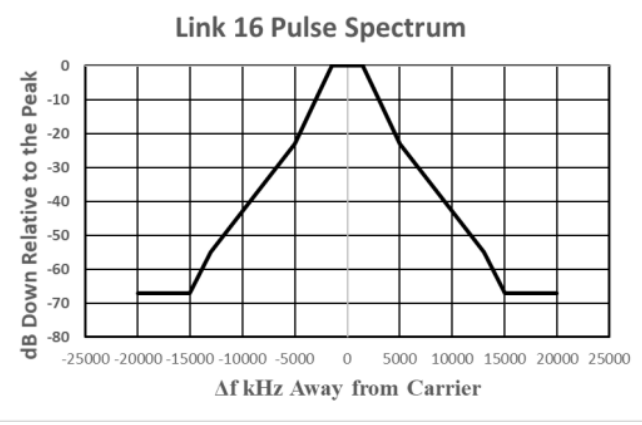

Fig. 11. JTIDS/MIDS Pulse Spectrum Model [1]

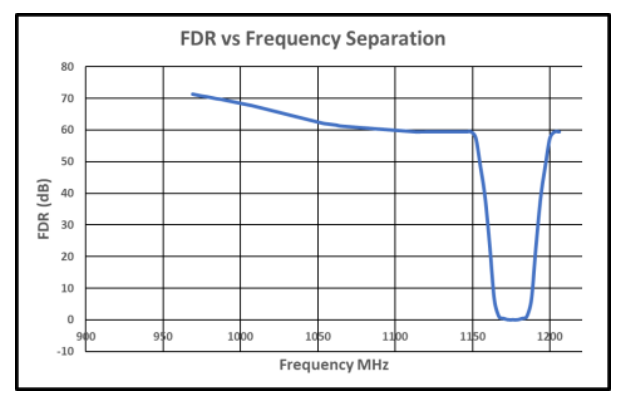

Fig. 12. Calculated FDR Coefficient Va1ues 


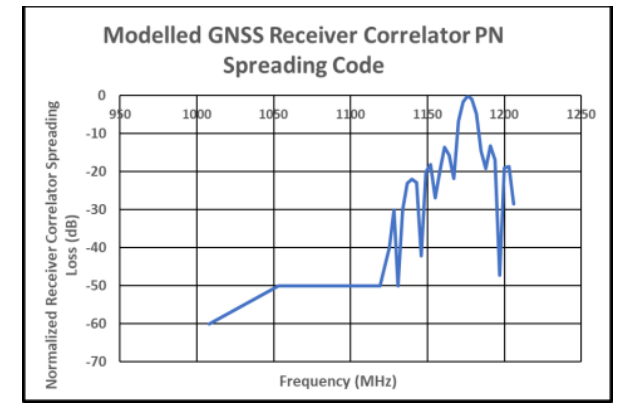

Fig. 13. Modelled GNSS Receiver Correlator PN Spreading Code

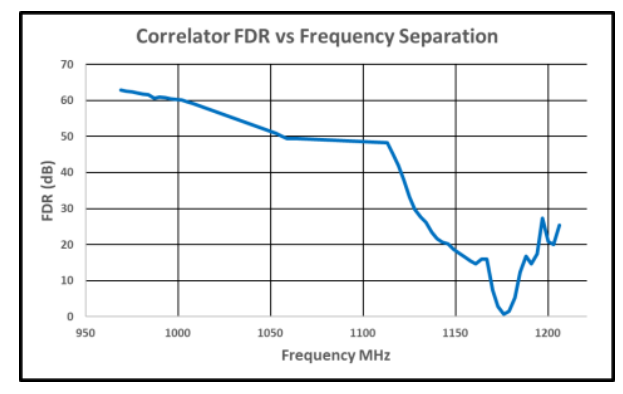

Fig. 14. Calculated SSC Values from FDR Analysis

TABLE VII. BDC CALCULATIONS PER MODELED JTIDS/MIDS POINT SOURCE

\begin{tabular}{|l|l|l|c|c|c|c|}
\hline \multicolumn{2}{|c|}{ Case } & Nc & FG & BG & Totals \\
\hline $\boldsymbol{8}$ & $\mathbf{8 A}$ & $\mathbf{5 0}$ NM APIS & 37 & 0.0562 & 0.0412 & 0.0975 \\
\hline $\boldsymbol{8}$ & $\mathbf{8 A}$ & $\mathbf{5 0} \boldsymbol{N M} \boldsymbol{A P I S}$ & 51 & 0.0407 & 0.0300 & 0.0707 \\
\hline
\end{tabular}

TABLE VIII. SUMMARY OF BDC AND RI WITH PULSE BLANKER THRESHOLD OF $-90 D B M$

\begin{tabular}{|l|c|c|c|c|c|c|}
\cline { 2 - 7 } \multicolumn{1}{c|}{} & \multicolumn{3}{c|}{37 carriers } & \multicolumn{3}{c|}{ 51 carriers } \\
\cline { 2 - 7 } \multicolumn{1}{c|}{} & $\boldsymbol{b d c}$ & $\boldsymbol{R}_{\boldsymbol{I}}$ & $\begin{array}{c}\boldsymbol{C} / \boldsymbol{N}_{\mathbf{0}} \\
\text { deg. }\end{array}$ & $\boldsymbol{b d c}$ & $\boldsymbol{R}_{\boldsymbol{I}}$ & $\begin{array}{c}\boldsymbol{C} / \boldsymbol{N}_{\mathbf{0}} \\
\text { deg. }\end{array}$ \\
\hline Case 8 & 0.0975 & 0.2759 & 1.50 & 0.0707 & 0.2001 & 1.11 \\
\hline Case 8A & 0.0975 & 0.5009 & 2.21 & 0.0707 & 0.3634 & 1.66 \\
\hline $\begin{array}{l}\mathbf{5 0} \mathbf{N M} \\
\text { APIS }\end{array}$ & 0.0975 & 0.6661 & 2.66 & 0.0707 & 0.4833 & 2.03 \\
\hline
\end{tabular}

TABLE IX. SUMMARY OF BDC AND RI WITH PULSE BLANKER THRESHOLD OF $-91 D B M$

\begin{tabular}{|l|c|c|c|c|c|c|}
\cline { 2 - 7 } \multicolumn{1}{c|}{} & \multicolumn{3}{c|}{37 carriers } & \multicolumn{3}{c|}{ 51 carriers } \\
\cline { 2 - 7 } \multicolumn{1}{c|}{} & $\boldsymbol{b d c}$ & $\boldsymbol{R}_{\boldsymbol{I}}$ & $\begin{array}{c}\boldsymbol{C} / \boldsymbol{N}_{\mathbf{0}} \\
\boldsymbol{d e g} .\end{array}$ & $\boldsymbol{b d c}$ & $\boldsymbol{R}_{\boldsymbol{I}}$ & $\begin{array}{c}\boldsymbol{C} / \boldsymbol{N}_{\mathbf{0}} \\
\boldsymbol{d e g} .\end{array}$ \\
\hline Case 8 & 0.0975 & 0.2244 & 1.32 & 0.0707 & 0.1628 & 0.97 \\
\hline Case 8A & 0.0975 & 0.3979 & 1.90 & 0.0707 & 0.2887 & 1.42 \\
\hline $\begin{array}{l}\mathbf{5 0} \text { NM } \\
\text { APIS }\end{array}$ & 0.0975 & 0.5504 & 2.35 & 0.0707 & 0.3993 & 1.78 \\
\hline
\end{tabular}

\section{Interfering scenarios $C / N_{0}$ degradation results}

The summary of $b d c$ and $R_{I}$ results for the Case 8, Case 8A and $50 \mathrm{NM}$ APIS case environments with a $-90 \mathrm{dBm}$ pulse blanker threshold for 37 or 51 carrier frequencies is shown in TABLE VII. The summary of the results with a $-91 \mathrm{dBm}$ pulse blanker threshold is shown in TABLE IX.

\section{CONCLUSIONS}

In this paper, the $C / N_{0}$ degradation of the main DO292 interfering scenario has been compared to the $C / N_{0}$ degradation of two new significant interfering scenario, 50NM APIS and case $8 \mathrm{~A}$ scenario. The largest degradation has been found for the 50 NM APIS scenario followed by case $8 \mathrm{~A}$ and case 8 . Results using the $-91 \mathrm{dBm}$ blanker threshold produced a lower $C / N_{0}$ degradation than the results using the $-90 \mathrm{dBm}$ blanker threshold. The same tendency was found between $20 \mathrm{MHZ}$ and $12 \mathrm{MHz}$ of RFFE filter bandwidth where a lower value implies more RFI signals attenuated by the filter and thus a lower $C / N_{0}$ degradation.

Presented $C / N_{0}$ degradation results were obtained through predictions and simulations. Prediction were done using [1] formulas and two new similar formulas for $R_{I}$, one proposed by ENAC in [3], and another one proposed by MITRE. Both ENAC and MITRE formulas predict a lower $C / N_{0}$ degradation than those previously published within the RTCA DO-292 document for Case 8; simulations confirmed the predictions using new $R_{I}$ formulas. Nevertheless, tighter predictions could be made by calculating the expected $b d c^{i}$ and filtered post-blanker PSD of the below-threshold pulses. Moreover, MITRE formula assumes that due to the unknown nature of the post blanker correlator frequency rejection response, the rejection will follow the PN spreading code which is a sinc 2 function. This is an area where the assumption needs to be confirmed by further studies.

Simulated results were obtained by generating equivalent JTIDS/MIDS pulses for interference analysis purposes. A description of the method used to generate the equivalent JTIDS/MIDS signals is provided.

\section{REFERENCES}

[1] RTCA, DO 292 - Assessment of RF interference relevant to the GNSS L5-E5a band, July 29, 2004

[2] A.Garcia-Pena et al., "Efficient DME/TACAN Blanking Method for GNSS-based Navigation in Civil Aviation," Proceedings of the 32nd ION GNSS+ 2019, Miami, Florida, September 2019, pp. 1438-1452.

[3] A.Garcia-Pena et al., "GNSS C/N0 Degradation Model in Presence of Continuous Wave and Pulsed Interference" Proceedings of the 2020 ITM, Reston, San Diego, January 2020.

[4] A. Garcia-Pena et al., "Impact of DME/TACAN on GNSS L5/E5a Receiver," Proceedings of the 2020 ITM, San Diego, California, January 2020, pp. 207-221.

[5] Gao, G. X., Heng, L., Hornbostel, A., Denks, H., Meurer, M., Walter, T., \& Enge, P. (2013). "DME/TACAN Interference Mitigation for GNSS: Algorithms and Flight Test Results". GPS Solutions, 17(4), 561-573

[6] Grabowski, J., \& Hegarty, C. (2002). "Characterization of L5 Receiver Performance Using Digital Pulse Blanking". Procedings of the Institute of Navigation GPS, (pp. 1630-1635). Portland, OR.

[7] Hegarty, C., Van Dierendonck, A. J., Bobyn, D., \& Grabowski, M. T. (2000). "Suppression of Pulsed Interference through Blanking". Proceedings of the IAIN World Congress and the 56th Annual Meeting of The Institute of Navigation , (pp. 399 - 408). San Diego, CA.

[8] Shallberg, K., Flake, J., Baraban, D., \& Hegarty, C. (2018). "Updated Aviation Assessment of Interference in the L5/E5A Bands from Distance Measuring Equipment". Proceedings of the 31st ION GNSS+ 2018, (pp. 1324-1337). Miami, Florida.

[9] T. Kim and J. Grabowski. "Validation of GPS L5 Coexistence with DME/TACAN and Link-16 Systems," Proceedings of the 16th ION GPS/GNSS 2003, Portland, OR, September 2003, pp. 1455-1469.

[10] Chi-han Kao, "Performance Analysis of a JTIDS/LINK-Type Waveform Transmitted over slow, flat Nakagami Fading Channels in the Presence of Narrowband Interference", Dissertation, 2008.

[11] Proakis J.G. and Salehi M., "Digital Communications - 5th Edition", McGrawhill International Edition, 2008 